\title{
CAMBIO TECNOLOGICO EN LA ADMINISTRACIÓN DE RIESGOS FINANCIEROS: EL CASO MEXICANO
}

\author{
Francisco Venegas-Martínez* \\ Centro de Investigación en Finanzas, Tecnológico de Monterrey, \\ Campus Ciudad de México \\ Jorge Miguel Carrillo Rivera \\ Director de la División de Negocios, Tecnológico de Monterrey, \\ Campus Ciudad de México
}

(Received 14 june 2001, accepted 6 september 2002)

\section{Resumen}

En este trabajo se presenta una estrategia integral para la administración de riesgos financieros sustentada en el cambio tecnológico. En México, el cambio tecnológico que demanda la administración de riesgos ha sido tímido, frágil y parcial, y en general está lejos de las expectativas de cambio del entorno. Las metodologías y aplicaciones disponibles para la administración de riesgos corresponden, en general, a paradigmas ajenos a nuestra realidad. I」a importación generalizada de técnicas y modelos sin las adaptaciones necesarias, no sólo ha mostrado ser ineficiente, sino que en ocasiones, ha generado mayor volatilidad y turbulencia en los mercados financieros. Con base en un diagnóstico detallado de la situación actual de la administración de riesgos en México, la conclusión principal de esta investigación es que los administradores de riesgos financieros tienen que estar comprometidos con el cambio tecnológico. Finalmente, se proporciona un conjunto de recomendaciones para las instituciones financieras en materia de administración de riesgos y cambio tecnológico.

\section{Abstract}

This paper presents an integral strategy for risk management sustained in technological change. In Mexico, technological change has been shy, fragile, and partial, and in general is far from the expectations of a changing environment. The methodologies and applications available in risk management are matched, in general, to paradigms different from our reality. The generalized adoption of techniques and models without the proper adaptation to our reality, has not only shown to be inefficient, but it has also generated greater volatility and turbulence in the financial markets. Based on of a detailed dignostic of the current situation of the risk management in Mexico, the main conclusion of this research is that risk managers should be committed with technological change. Finally, the paper provides a set of recommendations for financial institutions about risk management and technological change.

Clasificación JEL: G2, G13

Palabras clave: Cambio tecnológico, Administración de riesgos

* Centro de Invesgación de Finanzas, Tecnológico de Monterrey, Campus Ciudad de México, Calle del Puente 222, Aulas 3, Cuarto piso, Col. Ejidos de Huipulco, Del. Tlalpan, 14380 México, D. F., Teléfono: (52)54832254, Correo electrónico: fvenegas@itesm.mx

Los autores desean agradecer a dos dictaminadores anónimos sus valiosas observaciones. 


\section{Introducción}

En las últimas décadas los mercados financieros han experimentado profundas transformaciones sustentadas en el acelerado desarrollo de las tecnologías de información, facilitando con esto la operación y la diversificación de los portafolios de inversión. De la misma manera, el cambio tecnológico no sólo ha permitido reducir las distancias, sino también los tiempos. Así, la integración de los mercados financieros proporciona mayores alternativas de inversión con más y mejor información, reduciendo con ello la asimetría de lo que los agentes saben sobre el mercado. Este proceso no sólo ha modificado el comportamiento de los participantes, sino también las reglas del juego. Hoy, los instrumentos financieros se negocian en forma continua y la movilidad de capital tiende a ser perfecta.

En el marco de integración de los mercados financieros, la necesidad de cambio en el sistema financiero mexicano, como resultado de los avances en las tecnologías de información, se ha manifestado en una reforma estructural a través de tres vías distintas: 1) la apertura, para disminuir barreras sobre las operaciones financieras; 2) la desregulación, para adecuar la legislación de los mercados financieros; y 3) la administración de riesgos, para establecer medidas prudenciales para todos los participantes en el sistema financiero mexicano.

No obstante lo anterior, el cambio tecnológico que demanda la administración de riesgos ha sido, hasta la fecha, un cambio tímido, parcial y temporal, y en general no satisface las necesidades y expectativas de cambio en el entorno mundial. Los avances teóricos y prácticos de la administración de riesgos financieros generados en los países desarrollados han ampliado en forma espectacular los alcances en dicha disciplina. Sin embargo, las prioridades de investigación en estos países, así como las realidades económicas y financieras dentro de las que se realizan dichas investigaciones son muy diferentes a las prevalecientes en nuestro país. El sector financiero mexicano sigue adoptando modelos sin adaptarlos adecuadamente a nuestra realidad. Es necesario, por lo tanto, generar en el país una corriente de pensamiento rigurosa y sólida que proporcione las herramientas, modelos, técnicas y metodologías para la administración de riesgos financieros dentro del contexto nacional, así como dentro del nuevo orden de integración mundial de los mercados.

En este trabajo se presenta una estrategia integral para la administración de riesgos en el sistema financiero mexicano sustentada en el cambio tecnológico. Hasta ahora, el cambio tecnológico que demanda la administración de riesgos en el país deja mucho que desear. Las aplicaciones ("software") disponibles para la administración de riesgos corresponden, en general, a paradigmas ajenos a nuestra realidad. La constante importación de sistemas distantes a nuestro contexto, no sólo ha mostrado ser costosa, sino también ineficiente. ${ }^{1}$ Por lo tanto, es necesario desarrollar modelos $a d-h o c$ que reflejen las restricciones y necesidades de nuestras instituciones y mercados, los cuales tienen características únicas e irrepetibles.

1 Detalles sobre costos para el caso de México pueden verse en Venegas-Martínez y Dubcovsky (2002). 


\section{Los sistemas de administración de riesgos en México}

En México, al igual que en muchos otros países, la mayoría de las crisis financieras son el resultado del inadecuado manejo de los riesgos. Entre las principales causas de estos desastres, se encuentra la incipiente, y en ocasiones nula, práctica de medición de riesgos a los que se exponen las instituciones financieras. Basta recordar, los efectos negativos de la debacle financiera de diciembre de 1994, los cuales están todavía presentes en la banca comercial con un mercado de crédito interno deprimido y sin vistas de una pronta recuperación. En los últimos años, las autoridades financieras, han hecho un esfuerzo muy importante en materia de administración integral de riesgos, al emitir varias circulares sobre las reglas de carácter prudencial que todos los intermediarios están obligados a seguir.

Hay una máxima generalizada entre los constructores de sistemas que dice: "si entra basura, basura sale" ("garbage in, garbage out"). En el caso particular de los sistemas de administración de riesgos en México, esto podría ser una realidad. La mayoría de las instituciones carecen de sistemas integrales en donde la información de sus operaciones se realiza en tiempo real. En muchos casos, cada área de negocios tiene sistemas de "front office" especializados e independientes, por ejemplo uno pare el mercado de dinero, otro para derivados, uno más para cartera de crédito comercial, otro de cartera de crédito al consumo, etc. Adicionalmente, la base de datos de la contabilidad que consolida toda la información, no es analítica, ni está pensada para la administración de riesgos. Por lo anterior, uno de los primeros problemas para la implementación de nuevas tecnologías de administración de riesgos, es el acopio de los datos. Los riesgos operativos generados por estos problemas pueden incrementarse considerablemente, debido a la diferencia de tiempos entre lo que está sucediendo en las mesas de operación y los registros de dichas operaciones.

Existen muchos casos conocidos de sistemas de administración de riesgos que después de un gran esfuerzo por instalarlos, nunca se logra ponerlos a punto debido a las características tan particulares de las operaciones en nuestro país. Además de los múltiples problemas que se presentan en la interpretación de los resultados. Los sistemas disponibles, aunque parezca contradictorio, dificultan el proceso de monitoreo de riesgos, razón por la que muchas instituciones después de realizar inversiones de varios millones de dólares se decepcionan profundamente por la adquisición de tan costosas aplicaciones.

\section{Cambio tecnológico, "software" y administración de riesgos}

En las últimas décadas, la persistencia de volatilidad en los mercados financieros ha obligado a los sectores financieros de todo el mundo, a reconsiderar a la administración de riesgos como un asunto serio y prioritario, sobre todo en lo que se refiere a riesgos de mercado, crédito, operacional y país. Por ello, las instituciones financieras han gastado billones de dólares en la adquisición de nuevas tecnologías que les permitan monitorear y controlar eficientemente sus riesgos. A causa de este fenómeno, en la década de los noventa, se dio un desarrollo considerable en el "software" especializado para la.medición y 
control de riesgos financieros; basta mencionar que según la revista Derivatives Strategy se tienen listadas más de 130 aplicaciones distintas.

Por otra parte, cabe señalar que, en general, el "software" que requieren las instituciones financieras puede ser de gran complejidad, lo cual se comprende mejor al notar que en una institución el área de administración de riesgos ocupa el lugar de "middle-office", es decir, se encuentra entre el "front" y el "backoffice", esto obliga a que el "software" requerido en el control de riesgos sea muy versátil, ya que por un lado debe suministrar de información detallada a los operadores ("traders") de los niveles de riesgo que se están asumiendo, permitiéndoles monitorear sus operaciones de tal forma que no excedan los límites que fijan las políticas de la institución y, por otro lado, debe ser capaz de suministrar reportes adecuados a las áreas de "back-office" para que la información sea registrada en los libros de la institución. A su vez, el área de administración de riesgos debe tener acceso a distintos niveles de resumen para poder ubicar comportamientos atípicos de la cartera de la institución.

Otra característica que es deseable en el "software" para la medición de riesgos es la rapidez. Por lo general, el monitoreo del riesgo que un "trader" requiere debe ser preferentemente en tiempo real, lo cual obliga a mediciones frecuentes de riesgo $\mathrm{y}$, por ende, a que los sistemas de medición puedan suministrar datos intra-día, siendo esto una tarea difícil cuando el portafolio de la institución es de gran tamaño. Finalmente, otro aspecto que añade complejidad a un sistema eficiente de riesgos es que, por lo general, debe ser suficientemente flexible para interactuar con otros sistemas, que no necesariamente corren en la misma plataforma o que pueden ser administrados con manejadores de bases de datos de naturaleza distinta. De cualquier modo, y a pesar de todas las complejidades que reviste implementar un sistema de administración de riesgos, los beneficios esperados, cuando los procesos se llevan a cabo en forma adecuada, son considerables.

Dentro de la gran variedad de sistemas de administración de riesgos existentes en el mercado, los más sofisticados son las llamadas "suites" de "software" capaces de proporcionar datos de valor en riesgo calculados a través de cualquiera de los métodos tradicionales. Estos sistemas tienen un conjunto de funciones analíticas para la generación de escenarios y pruebas de "stress". Mediante el uso de los modelos especificados, los sistemas disponibles pueden valuar los instrumentos de un determinado portafolio, así como evaluar distintas medidas de riesgo con base, fundamentalmente, en el conjunto de escenarios que se suministraron. Estas aplicaciones tienen la capacidad para resumir las diferentes posiciones de un portafolio, lo cual da lugar a la generación de reportes con diferentes niveles de detalle. Otra de sus características es la rapidez con la que proporcionan las medidas que se le solicitan, aún con portafolios de magnitud considerable. También, estas aplicaciones tienen la capacidad de manejar posiciones en distintas divisas y de muy diversos tipos de instrumentos. Sin embargo, estos paquetes tienen como limitación el que no son sencillos de instalar. Adicionalmente, resultan poco amigables con el usuario y rígidos al momento de instrumentar cambios profundos en los modelos predeterminados. 


\section{Modelos y patrones}

Los avances teóricos en la administración de riesgos financieros han fomentado el uso de aplicaciones con herramientas sofisticadas como: el cálculo estocástico y el control óptimo estocástico. Así mismo, la mayor parte de la literatura financiera moderna tiene un contenido importante de dichas herramientas, siendo el rigor matemático la tendencia que prevalece en la investigación financiera. En este marco, el patrón típico en el modelado del comportamiento de una variable financiera, $X(t)$, corresponde a un proceso Markoviano de difusión (véase, por ejemplo, Venegas-Martínez, 2001):

$$
\mathrm{d} X(t)=\mu[t, X(t), U(t)] \mathrm{d} t+\sigma[t, X(t), U(t)] \mathrm{d} W(t),
$$

donde $\mu[t, X(t), U(t)]$ y $\sigma[t, X(t), U(t)]$ son funciones conocidas, y $W(t) \sim$ $\mathcal{N}(0, \mathrm{~d} t)$ es un proceso de Wiener. De la misma forma, a través de la función $U(t)$ es posible modelar peculiaridades del entorno financiero, incluyendo aspectos ideosincráticos. Mientras que el patrón típico en los problemas de toma de decisiones de los agentes financieros es:

$$
\begin{gathered}
\text { Maximizar } \int_{0}^{\infty} e^{-\rho t} F(U(t), X(t)) \mathrm{d} t, \\
\text { sujeto a : } \mathrm{d} X(t)=\mu[t, X(t), U(t)] \mathrm{d} t+\sigma[t, X(t), U(t)] \mathrm{d} W(t), \\
U(t) \in \mathcal{U},
\end{gathered}
$$

donde $U(t)$ es la variable de control, $X(t)$ es la variable de estado, $\rho$ es un factor de descuento (que puede depender del tiempo), y $\mathcal{U}$ es el conjunto de controles congruentes (factibles o admisibles) con la realidad económica y financiera de un país. Por lo tanto, el planteamiento, la intuición e interpretación de los resultados derivados de los patrones, establecidos en (1) y (2), deben tomar en cuenta las restricciones, tan particulares, que impone nuestro sistema financiero.

Por otro lado, una de las principales características que distingue a los mercados emergentes es la presencia de movimientos atípicos e inesperados (auges o caídas) en los precios de los activos. Estos movimientos ocurren con mayor frecuencia de lo que se esperaría bajo el supuesto de una distribución normal (cuando se estudian rendimientos), incluso con una volatilidad razonablemente moderada. En el análisis de datos financieros, cuando se compara una distribución estandarizada empírica con una distribución normal estándar, se observa con frecuencia que la cresta de la distribución empírica es más alta. Dado que ambas distribuciones tienen la misma desviación estándar, es decir, los mismos puntos de inflexión, entonces las colas de la distribución empírica tienen necesariamente que ser más anchas para compensar el área de la cresta, que en ambos casos tiene que ser igual a uno. La cresta más alta significa que existe una probabilidad más grande de movimientos pequeños que la de una variable aleatoria con distribución normal. Además, debido a las colas más gordas (o pesadas) de la distribución empírica, se tiene una mayor probabilidad de que ocurran valores extremos en comparación con la distribución normal. En este caso, si la distribución nornal se mezcla con una distribución que genere 
movimientos extremos en los rendimientos, se producirán colas más pesadas y se tendría una mejor aproximación a lo observado.

En conclusión, el desarrollo de sistemas integrales de administración de riesgos debe considerar el conjunto $\mathcal{U}$ que mejor represente el entorno financiero y el modelo que mejor describa la dinámica de las variables financieras en el marco macroeconómico del país. De esta manera, la valuación de derivados y notas estructuradas, así como la cobertura de riesgos con base en estos instrumentos serán más confiables. Específicamente, se supone que la dinámica de la variable financiera corresponde a (un proceso mixto de difusión con saltos):

$$
\mathrm{d} X(t)=\mu[t, X(t), U(t)] \mathrm{d} t+\sigma[t, X(t), U(t)] \mathrm{d} W(t)+\nu[t, X(t), U(t)] \mathrm{d} N(t),
$$

donde $N(t)$ es un proceso de Poisson con media $\lambda$ d $t$. Aquí, $\lambda$ es el parámetro de intensidad y $\nu[t, X(t), U(t)]$ es el tamaño esperado del salto, el cual puede ser determinista o estocástico.

Otra posibilidad para modelar exceso de curtosis, colas pesadas e incluso sesgo, es a través de modelos de volatilidad estocástica con reversión en la media

$$
\begin{gathered}
\mathrm{d} X(t)=\mu[t, X(t), U(t)] \mathrm{d} t+\sigma[t, X(t), U(t)] \mathrm{d} W(t), \\
\mathrm{d} \sigma^{2}[t, X(t), U(t)]=\alpha\left(\beta-\sigma^{2}[t, X(t), U(t)]\right) \mathrm{d} t+s[t, X(t), U(t)] \mathrm{d} V(t),
\end{gathered}
$$

donde $\alpha$ y $\beta$ son parámetros por estimar, $s[t, X(t), U(t)]$ es conocida y $V(t) \sim$ $\mathcal{N}(0, \mathrm{~d} t)$. Los procesos $W(t)$ y $V(t)$ podrían estar correlacionados.

\section{Nuevos paradigmas en la administración de riesgos}

Dentro del campo de la física, bajo el supuesto de sistemas inerciales y bajo condiciones de espacio y tiempo absoluto, las leyes de la mecánica Newtoniana. describen la trayectoria de una partícula, o un sistema de partículas. Sin embargo, estas leyes no son capaces de explicar los movimientos de las partículas atómicas. En respuesta a la necesidad de contar con paradigmas más realistas, la física moderna, en particular, la mecánica estadística y la mecánica cuántica han incorporado nuevos conceptos teóricos como: relatividad general, incertidumbre, inestabilidad y caos. En el campo de las finanzas, una historia parecida es aplicable. En la búsqueda de modelos que expliquen en forma más realista el comportamiento de los mercados bajo condiciones de incertidumbre, la administración de riesgos financieros ha comenzado a desechar modelos "actuariales" (mecanicistas) y fomentado el uso de los procesos de difusión combinados con procesos de saltos de Poisson como herramientas (cuánticas) más adecuadas para el estudio y control de situaciones de riesgo. Si bien, estas herramientas son indispensables, no son suficientes para una administración adecuada y oportuna de riesgos si no se complementan con un cambio tecnológico. Para ilustrar esta situación, considere la ecuación de Schrödinger de la función de onda unidimensional, $u(x, \tau)$, la cual está dada por:

$$
A \frac{\partial u}{\partial \tau}=\frac{\partial^{2} u}{\partial x^{2}}, \quad \text { para } \quad-\infty<x<\infty, \quad \tau>0,
$$


donde $A$ es una constante. La ecuación (5) es fundamental en mecánica cuántica para el estudio de la distribución de probabilidad espacial (unidimensional) de una partícula. Por otro lado, considere la ecuación diferencial parcial parabólica de segundo orden de Black-Scholes y las condiciones de frontera para un "call" europeo, $c(S, t)=\max (S-K, 0)$ :

$$
\frac{\partial c}{\partial S}+\frac{1}{2} \sigma^{2} S^{2} \frac{\partial^{2} c}{\partial S^{2}}+r S \frac{\partial c}{\partial S}-r c=0
$$

con

$$
c(0, t)=0 \quad \text { y } \quad c(S, t) \approx S \quad \text { cuando } \quad S \rightarrow \infty .
$$

Mediante los cambios de variable,

$$
S=K e^{x} \quad \text { y } \quad t=T-\frac{2 \tau}{\sigma^{2}},
$$

se tiene que

$$
c(S, t)=K \nu(x, \tau)
$$

Si además,

$$
\nu(x, \tau)=e^{\alpha x+\beta \tau} u(x, \tau),
$$

donde $\alpha$ y $\beta$ se tienen que determinar, se cumple que la función $u$ satisface la ecuación

$$
B \frac{\partial u}{\partial \tau}=\frac{\partial^{2} u}{\partial x^{2}}, \quad \text { para }-\infty<x<\infty, \quad \tau>0,
$$

donde $B$ es una constante. En este caso, además se requieren condiciones iniciales congruentes con (6). La similitud entre las ecuaciones (6) y (7), no es simple coincidencia, ya que las fluctuaciones en los precios y el movimiento de las partículas forman parte del mismo universo y, por ende, regidos por las mismas leyes. En conclusión, se requieren cambios de actitud y de tecnología más "cuánticos" en la forma de abordar nuestros problemas de administración de riesgos.

Dentro de este gran marco conceptual, se requieren cambios que nos lleven a desechar conceptos mecanicistas incongruentes con el mundo cuántico de la administración de riesgos. Algunas de las posturas, "mecanicistas" en un sentido más amplio, con las que frecuentemente nos enfrentamos y que se tienen que desechar son las siguientes:

1) "Nuestra institucion financiera ha dado un giro de 180 grados en la admillistración de riesgos"... este cambio probablemente ha implicado un cambio de dirección, pero con la misma tecnología.

2) "Hemos invertido millones de dólares en un sistema de administración de riesgos con la más alta capacidad de procesamiento de datos"... el gran problema de las instituciones financieras no es el volumen de información existente, sino la relevancia y disponibilidad de la información.

3) "En esta institución financiera todas la reglas sobre la administración de riesgos están escritas, y los procedimientos son exhaustivos; todo está bajo 
control"... sería quizás más interesante conocer cuales son las reglas no escritas, y verificar si realmente los procedimientos son capaces de detectar situaciones inesperadas.

4) "Lo más valioso de esta institución financiera es la experiencia que tiene en la administración de riesgos"... esto puede ser también su mayor debilidad, si $n o$ se llevan a cabo los cambios tecnológicos que demanda el entorno.

\section{Crisis financieras: catalizadores del cambio}

De 1975 a la fecha, el Fondo Monetario Internacional ha contabilizado más de 170 crisis monetarias y 60 bancarias, muchas de las cuales se suscitaron de manera simultanea, y en la mayoría de las ocasiones agravadas por crisis cambiarias. $^{2}$ Las crisis obligan frecuentemente a los participantes en el sector financiero a instrumentar cambios que antes les parecían imposibles. Las crisis son catalizadores de transformación, pero el cambio obligado difiere profundamente del cambio planificado. En el primer caso, se toman medidas de emergencia de corto plazo y las acciones están encaminadas a la supcrvivencia de la institución financiera. Mientras que en el segundo caso, se desea alcanzar la permanencia de la institución competitiva. En conclusión, la crisis provoca el cambio, pero un cambio completamente sufrido, a menudo demasiado tarde, y sin visión de largo plazo ya que las instituciones buscan recuperar lo que se puede salvar del pasado, en vez de construir el porvenir. Por lo anterior, como medida preventiva para enfrentar las crisis es importante la planeación financiera oportuna en la administración de riesgos, la cual tiene que ir más allá de las medidas de corto plazo. En particular, el uso de los productos derivados permite la planeación de largo plazo de los activos y pasivos de las instituciones financieras.

La crisis financiera mexicana de 1995 , de la cual todavía quedan razagos en el sector bancario, nos invita a evaluar sus lamentables consecuencias en la mediana y pequeña empresa por no haber tomado las medidas necesarias para cubrirse del riesgo de tasa de interés o de tipo de cambio. En México se cuenta con un mercado de productos derivados, el MexDer, altamente organizado y reconocido por las autoridades fiscales y financieras, donde se cotizan y negocian contratos a futuro estandarizados. Con un mercado estandarizado de derivados, la exposición a los distintos riesgos financieros puede reducirse considerablemente si se toman adecuada y oportunamente posiciones sobre estos instrumentos.

\section{Derivados como seguros contra contingencias financieras}

Como parte de los esfuerzos en la supervisión prudencial sobre las operaciones de las instituciones financieras, destaca la administración de riesgos mediante el uso de productos derivados. Las instituciones financieras toman posiciones

2 Entre las principales causas que influyen en la generación de una crisis se encuentran: a) contagio de volatilidad por la globalización de los mercados financieros; b) cambios abruptos en las preferencias de los inversionistas; y c) problemas sociales y politicos del país o región. 
con derivados, largas o cortas y por cierto tiempo, en contratos a plazo ("forwards"), permutas ("swaps"), opciones y notas estructuradas para cubrir riesgos de crédito, mercado, liquidez, operacional, legal, etcétera.

Los productos derivados son utilizados por las instituciones financieras de todo el mundo como herramientas de administración de riesgos. Estos instrumentos permiten identificar, cuantificar y aislar distintos tipos de riesgos. Cuando estos instrumentos son utilizados oportunamente pueden reducir la exposición a los distintos riesgos. Los derivados pueden ser también usados para reducir costos financieros o para incrementar el rendimiento de ciertos activos. Para muchas instituciones financieras, los productos derivados producen una fuente directa de rentas a través del proceso "mark to market".

El tamaño considerable que han alcanzado los mercados de derivados financieros se debe, en gran medida, a la flexibilidad que estos instrumentos proporcionan a sus usuarios para entrar o salir rápidamente del mercado debido a la liquidez que generan (i.e., siempre es posible encontrar compradores y vendedores) y al apalancamiento que presentan (i.e., la inversión inicial es pequeña comparada con la de otros instrumentos). Los derivados son herramientas útiles que permiten a los inversionistas administrar el riesgo de mercado con bajos costos de transacción. Además, cuando las transacciones se llevan a cabo con derivados listados (negociados y cotizados en bolsas), el riesgo contraparte es mínimo debido a la asociación del mercado con una cámara de compensación y liquidación que garantiza el cumplimiento de las obligaciones adquiridas en los contratos.

A continuación se presenta un conjunto de técnicas de cobertura de riesgos de tasas de interés y de tipo de cambio con derivados. La característica principal de estas técnicas es que son congruentes con nuestra realidad económica y financiera.

\subsection{Cobertura de tasa de interés con productos derivados}

El riesgo por fluctuaciones adversas en la tasa de interés que enfrentan las instituciones financieras se traduce en el riesgo de que el valor presente de los flujos financieros, activos y/o pasivos, que se tienen planeados no se presente en la magnitud que se esperaba, lo que afecta la programación de las decisiones de gasto, inversión y financiamiento. El riesgo de tasas de interés puede reducirse si se cubre adecuadamente el valor presente de los flujos esperados, tomando posiciones sobre productos derivados de tasas.

El supuesto de que las tasas de interés se mantienen constantes o bien que su dinámica está determinada por una función conocida en el tiempo, podría ser razonable para instrumentos de renta fija de muy corto plazo, digamos días o posiblemente semanas, en períodos de estabilidad. Sin embargo, en el mediano y largo plazo, la tasa de interés presenta un comportamiento aleatorio, en cuyo caso el movimiento Browniano proporciona una herramienta adecuada 
de análisis. Específicamente, se supone que la tasa ("spot") o tasa instantánea de interés, $r_{t}$, es conducida por la siguiente ecuación diferencial estócastica:

$$
\mathrm{d} r_{t}=\mu\left(r_{t}, t\right) \mathrm{d} t+\sigma\left(r_{t}, t\right) \mathrm{d} W_{t},
$$

en donde $\mu\left(r_{t}, t\right)$ y $\sigma\left(r_{t}, t\right)$ son funciones conocidas. El proceso estocástico $W_{t}$ es un proceso de Wiener estandarizado, es decir, $W_{t}$ es una variable aleatoria normal con incrementos temporales independentes, la cual satisface $\mathrm{E}\left[\mathrm{d} W_{t}\right]=0$ $\mathrm{y} \operatorname{Var}\left[\mathrm{d} W_{t}\right]=\mathrm{d} t$. Las formas funcionales $\mu\left(r_{t}, t\right)$ y $\sigma\left(r_{t}, t\right)$ tienen que ser escogidas de tal forma que representen la dinámica de las tasas de interés en nuestra realidad financiera ( $v . g$. un mercado primario de subasta de títulos gubernamentales, un mercado secundario determinado por las necesidades de liquidez de nuestras instituciones financieras, un mercado de títulos de regulación monetaria, etcétera.).

A continuación se presenta la ecuación de compensación entre flujos financieros y derivados de tasas. Vamos a denotar al proceso que conduce al precio de un producto derivado de tasa de interés mediante $D\left(r_{t}, t ; T\right)$, donde $r_{t}$ es la tasa "spot", $t$ es la fecha de emisión del instrumento y $T$ es la fecha de vencimiento. Para inmunizar el valor presente de un conjunto de flujos de efectivo, se utilizarán tres productos derivados con vencimientos $T_{1}, T_{2}$ y $T_{3}, T_{1}<T_{2}<T_{3}$. Así pues, el precio del derivado con vencimiento $T_{i}$ es $D_{i}\left(r_{t}, t ; T_{i}\right), i=1,2,3$. Considere una estrategia de cobertura con $x_{i}$ unidades de cada derivado con vencimiento en $T_{i}$. Sea $V\left(r_{t}, t ; f\right)$ el valor presente de un conjunto de flujos de efectivo, $f=\left\{f_{1}, f_{2}, \ldots, f_{n}\right\}$, cada uno de los cuales se presenta en una fecha preestablecida $\tau_{j}, j=1,2, \ldots, n$. Se desean determinar cantidades $x_{1}, x_{2}$ y $x_{3}$ de tal manera que los cambios en el valor presente de $f, V\left(r_{t}, t ; f\right)$, por variaciones en la tasa de interés se compensen con los flujos generados por los derivados con distintos vencimientos, es decir,

$$
0=x_{1} \mathrm{~d} D_{1}+x_{2} \mathrm{~d} D_{2}+x_{3} \mathrm{~d} D_{3}+\mathrm{d} V .
$$

Si $\mathrm{d} V<0$, por lo menos un $\mathrm{d} D_{i}$ es positivo y, recíprocamente, si $\mathrm{d} V>0$, por lo menos un $\mathrm{d} D_{i}$ es negativo. La aplicación del Lema de Itô a $D_{i}\left(r_{t}, t ; T_{i}\right)$, $i=1,2,3$, conduce $\mathrm{a}^{3}$ :

$$
\begin{aligned}
0= & x_{1} \mathrm{~d} D_{1}+x_{2} \mathrm{~d} D_{2}+x_{3} \mathrm{~d} D_{3}+\mathrm{d} V \\
= & x_{1}\left[\left(\frac{\partial D_{1}}{\partial t}+\mu\left(r_{t}, t\right) \frac{\partial D_{1}}{\partial r_{t}}+\frac{1}{2} \sigma\left(r_{t}, t\right)^{2} \frac{\partial^{2} D_{1}}{\partial r_{t}^{2}}\right) \mathrm{d} t+\sigma\left(r_{t}, t\right) \frac{\partial D_{1}}{\partial r_{t}} \mathrm{~d} W_{t}\right] \\
& +x_{2}\left[\left(\frac{\partial D_{2}}{\partial t}+\mu\left(r_{t}, t\right) \frac{\partial D_{2}}{\partial r_{t}}+\frac{1}{2} \sigma\left(r_{t}, t\right)^{2} \frac{\partial^{2} D_{2}}{\partial r_{t}^{2}}\right) \mathrm{d} t+\sigma\left(r_{t}, t\right) \frac{\partial D_{2}}{\partial r_{t}} \mathrm{~d} W_{t}\right] \\
& +x_{3}\left[\left(\frac{\partial D_{3}}{\partial t}+\mu\left(r_{t}, t\right) \frac{\partial D_{3}}{\partial r_{t}}+\frac{1}{2} \sigma\left(r_{t}, t\right)^{2} \frac{\partial^{2} D_{3}}{\partial r_{t}^{2}}\right) \mathrm{d} t+\sigma\left(r_{t}, t\right) \frac{\partial D_{3}}{\partial r_{t}} \mathrm{~d} W_{t}\right] \\
& +\left(\frac{\partial V}{\partial t}+\mu\left(r_{t}, t\right) \frac{\partial V}{\partial r_{t}}+\frac{1}{2} \sigma\left(r_{t}, t\right)^{2} \frac{\partial^{2} V}{\partial r_{t}^{2}}\right) \mathrm{d} t+\sigma\left(r_{t}, t\right) \frac{\partial V}{\partial r_{t}} \mathrm{~d} W_{t} .
\end{aligned}
$$

3 Una discusión básica sobre el Lema de Itô puede verse, por ejemplo, en Venegas-Martínez (2001a). 
Después de reagrupar términos en $\mathrm{d} t \mathrm{y} \mathrm{d} r_{t}$, se obtiene que:

$$
\begin{aligned}
0= & \left(x_{1} \frac{\partial D_{1}}{\partial t}+x_{2} \frac{\partial D_{2}}{\partial t}+x_{3} \frac{\partial D_{3}}{\partial t}+\frac{\partial V}{\partial t}\right) \mathrm{d} t \\
& +\mu\left(r_{t}, t\right)\left(x_{1} \frac{\partial D_{1}}{\partial r_{t}}+x_{2} \frac{\partial D_{2}}{\partial r_{t}}+x_{3} \frac{\partial D_{3}}{\partial r_{t}}+\frac{\partial V}{\partial r_{t}}\right) \mathrm{d} r_{t} \\
& +\frac{1}{2} \sigma\left(r_{t}, t\right)^{2}\left(x_{1} \frac{\partial^{2} D_{1}}{\partial r_{t}^{2}}+x_{2} \frac{\partial^{2} D_{2}}{\partial r_{t}^{2}}+x_{3} \frac{\partial^{2} D_{3}}{\partial r_{t}^{2}}+\frac{\partial^{2} V}{\partial r_{t}^{2}}\right) \mathrm{d} t
\end{aligned}
$$

Dado que $\mathrm{d} t \mathrm{y} \mathrm{d} W_{t}$ son variables independientes en (10), entonces las tres condiciones siguientes:

$$
\left\{\begin{array}{l}
x_{1} \frac{\partial D_{1}}{\partial t}+x_{2} \frac{\partial D_{2}}{\partial t}+x_{3} \frac{\partial D_{3}}{\partial t}+\frac{\partial V}{\partial t}=0 \\
x_{1} \frac{\partial D_{1}}{\partial r_{t}}+x_{2} \frac{\partial D_{2}}{\partial r_{t}}+x_{3} \frac{\partial D_{3}}{\partial r_{t}}+\frac{\partial V}{\partial r_{t}}=0 \\
x_{1} \frac{\partial^{2} D_{1}}{\partial r_{t}^{2}}+x_{2} \frac{\partial^{2} D_{2}}{\partial r_{t}^{2}}+x_{3} \frac{\partial^{2} D_{3}}{\partial r_{t}^{2}}+\frac{\partial^{2} V}{\partial r_{t}^{2}}=0
\end{array}\right.
$$

implican la relación establecida en (10) entre los precios de los derivados y el valor presente de los flujos de efectivo ( $c f$. Venegas-Martínez, 2002). El sistema anterior tiene tres ecuaciones en las incógnitas $x_{1}, x_{2}$ y $x_{3}$. Se supone, por supuesto, que el determinante asociado al sistema (11) es distinto de cero a fin de garantizar soluciones no triviales. Los valores $x_{1}, x_{2}$ y $x_{3}$ definen una estrategia de cobertura. Las cantidades $\partial D_{i} / \partial r_{t}$ y $\partial^{2} D_{i} / \partial r_{t}^{2}, i=1,2,3$, se conocen como duración y convexidad monetarias del derivado $D_{i}$, respectivamente. De igual manera, la sensibilidad del valor presente a cambios en la tasa de interés, $\partial \mathrm{V} / \partial r_{t}$ recibe el nombre de duración monetaria de $V$ y la cantidad $\partial^{2} V / \partial r_{t}^{2}$ es llamada convexidad monetaria de $V$. Note que la duración se determina a través de una aproximación lineal, o de primer orden, entre un valor presente y la tasa de interés. La duración mide también el tiempo promedio (ponderado por el valor presente de los flujos) en que se presentan los flujos. Las cantidades $\partial D_{i} / \partial t$, $i=1,2,3$, y $\partial \mathrm{V} / \partial t$ miden la sensibilidad de los derivados y del valor presente a cambios en la fecha de inicio de la cobertura, respectivamente.

La experiencia en México demuestra que los cambios en la estructura de plazos no son pequeños desplazamientos, es decir, la relación que se presenta entre el valor presente de los flujos y la tasa de interés no es lineal. En consecuencia, es importante considerar una medida de sensibilidad de segundo orden conocida como convexidad. La convexidad es una medida de dispersión, con respecto de la duración, de los tiempos en que se presentan los flujos, entre mayor sea la convexidad más grande será la dispersión entre dichos tiempos. La agregación de la convexidad a la duración, nos permite tener una medida más precisa del cambio en el valor presente de los flujos esperados ante variaciones moderadas en la tasa de interés. Como puede observarse, la inmunización por duración y convexidad es fundamentalmente un método local, aplicable en el instante $\mathrm{d} t$, y diseñado para cubrir cambios en el valor presente de un conjunto de 
flujos de efectivo por desplazamientos paralelos y pequeños en la tasa de interés. Por esta razón, las estrategias, $x_{i}, i=1,2,3$, de inmunización requieren de actualizaciones periódicas o rebalanceo (resolver de nuevo el sistema (11)) a fin de proteger eficazmente no sólo contra pequeños desplazamientos en las tasas, sino también contra cambios moderados. Finalmente, dado el comportamiento de la estructura de plazos de la tasa de interés en México, es importante que los sistemas de administración de riesgos de tasas incluyan siempre medidas de segundo orden (convexidad) a fin de cuantificar y monitorear adecuadamente los riesgos.

\subsection{Cobertura de tipo de cambio con futuros}

La pregunta que se responde en esta sección, es cómo podríamos cubrir flujos financieros denominados en dólares para evitar que se generen pérdidas en su valor nominal por la exposición al riesgo mercado. Para llevar a cabo la cobertura de flujos de efectivo que se tienen programados podríamos seguir los tres principios siguientes:

1) Tomar una posición con futuros inversa a la posición que se mantiene sobre el flujo. Es decir, si estamos largos en nuestros flujos, entonces tomamos una posición corta en futuros y viceversa.

2) Determinar el número de contratos sobre los que necesitamos abrir posiciones en futuros. Esto lo podríamos llamar "ajuste por volumen".

3) Rebalancear periódicamente las posiciones de los contratos a futuro a medida que se mueva el tipo de cambio.

El valor nominal del portafolio combinado de flujos de efectivo y de futuros utilizados como cobertura en la fecha base, digamos la fecha $n$, denotado por $V^{(n)}=V^{(n)}(f, F)$, está dado por:

$$
V^{(n)}=S^{(n)} \sum_{l=1}^{m} \frac{1+r_{l, M}^{(n)}\left[\frac{t_{l}}{360}\right]}{1+r_{l, E}^{(n)}\left[\frac{t_{l}}{360}\right]} f_{l}+\sum_{i=1}^{3} G x_{i}\left[F_{t, T_{i}}^{(n)}-S^{(n)} \frac{1+r_{T_{i}, M}^{(n)}\left[\frac{T_{i}}{360}\right]}{1+r_{T_{i}, E}^{(n)}\left[\frac{T_{i}}{360}\right]}\right],
$$

donde $f=\left\{f_{1}, f_{2}, \ldots, f_{l}, \ldots, f_{m}\right\}$ son flujos de efectivo en dólares (si $f_{l}<0$ el flujo es un pasivo, si $f_{l}>0$ es un activo). Las fechas en que ocurren los flujos son preestablecidas y se denotan por $t_{1}, t_{2}, \ldots, t_{l}, \ldots, t_{m}$. Asimismo, $F=$ $\left\{F_{t, T_{1}}^{(n)}, F_{t, T_{2}}^{(n)}, F_{t, T_{3}}^{(n)}\right\}$ son tres series de contratos a futuro de dólar con fechas de vencimiento $T_{1}, T_{2}$ y $T_{3}$. La cantidad $G$ representa el tamaño del contrato. También, $r_{l, M}^{(n)}$ denota la tasa de interés en la economía doméstica de plazo $l, r_{l, E}^{(n)}$ representa la tasa de interés en la economía extranjera de plazo $l, \mathrm{y}$ $S^{(n)}$ es el tipo de cambio nominal, todos ellos referenciados a la fecha base $n$. Observe que en la primera sumatoria se determina el valor presente de los flujos en dólares, en términos de pesos, llevados a valor futuro (momento en que se dan estos flujos) dado el tipo de cambio y las tasas de interés domésticas actuales. En la segunda sumatoria se expresan los flujos que se generan con los 
contratos futuros en los tiempos $T_{i}$. Es importante destacar que conforme nos aproximamos a $T_{i}$ el precio del futuro se aproxima al precio "spot" del dólar. Por esta razón, se presenta un flujo de efectivo por la operación pactada (compra o venta). Note que ambos flujos tienen signos contrarios ya que si la operación a futuro es larga (compra) entonces en $T_{i}$ se paga el precio pactado del dólar. En el caso de una posición corta (venta), el razonamiento es inverso. Esto es precisamente lo que genera los flujos compensatorios que cubren al portafolio. ${ }^{4}$

Una vez que se ha establecido la manera de cuantificar la magnitud de los cambios en el valor nominal de los flujos de efectivo y en los futuros debidos a variaciones en el tipo de cambio y en la tasa de interés, estamos en condiciones de determinar el número de contratos y la posición que se requiere (larga $o$ corta) para compensar los cambios en el portafolio combinado. El método que se propone para cubrir los flujos financieros denominados en dólares es como sigue: se determinan las sensibilidades del valor nominal del portafolio combinado (flujos y futuros) a variaciones en el tipo de cambio y en las tasas de interés, y posteriormente se igualan a cero, de tal manera que los cambios en el valor nominal de los flujos por variaciones en el tipo de cambio y en las tasas de interés, se compensen con los flujos generados por los contratos futuros. E1 sistema resultante contempla tres ecuaciones con tres incógnitas, digamos $x_{1}, x_{2}$ y $x_{3}$, que representan el número de contratos a futuro sobre dólar de tres series. Así pues, a partir de (12), el sistema que se tiene que resolver está dado por:

$$
\begin{gathered}
\frac{\partial V^{(n)}}{\partial S^{(n)}}=\sum_{l=1}^{m} \frac{1+r_{l, M}^{(n)}\left[\frac{t_{l}}{360}\right]}{1+r_{l, E}^{(n)}\left[\frac{t_{l}}{360}\right]} f_{l}-\sum_{i=1}^{3} G x_{i} \frac{1+r_{T_{i}, M}^{(n)}\left[\frac{T_{i}}{360}\right]}{1+r_{T_{i}, E}^{(n)}\left[\frac{T_{i}}{360}\right]}=0, \\
\frac{\partial V^{(n)}}{\partial r_{l, M}^{(n)}}=S^{(n)} \sum_{l=1}^{m} \frac{\left[\frac{t_{l}}{360}\right]}{1+r_{l, E}^{(n)}\left[\frac{t_{L}}{360}\right]} f_{l}-S^{(n)} \sum_{i=1}^{3} G x_{i} \frac{\left[\frac{r_{i}}{360}\right]}{1+r_{T_{i}, E}^{(n)}\left[\frac{T_{i}}{360}\right]}=0, \\
\frac{\partial V^{(n)}}{\partial r_{l, E}^{(n)}}=-S^{(n)} \sum_{l=1}^{m} \frac{\left[1+r_{l, M}^{(n)}\left[\frac{t_{l}}{360}\right]\right]\left[\frac{t_{L}}{360}\right]}{\left[1+r_{l, E}^{(n)}\left[\frac{t_{l}}{360}\right]\right]^{2}} f_{l} \\
+S^{(n)} \sum_{i=1}^{3} G x_{i} \frac{\left[1+r_{T_{i}, M}^{(n)}\left[\frac{T_{i}}{360}\right]\right]\left[\frac{T_{i}}{360}\right]}{\left[1+r_{T_{i}, E}^{(n)}\left[\frac{T_{i}}{360}\right]\right]^{2}}=0 .
\end{gathered}
$$

Observe que $x_{1}, x_{2}$ y $x_{3}$ dependen de varios factores: 1 ) de los montos y fechas de los flujos de efectivo en dólares y 2) de los precios y vencimientos de los contratos futuros. Si $x_{i}>0$, se genera una posición larga (posición de compra en contratos), en caso contrario se genera una posición corta (posición de venta en los contratos). El costo de la estrategia de cobertura se calcula multiplicando

4 Véanse, por ejemplo, González-Arechiga, Venegas-Martínez y Díaz Tinoco (2000), e Islas Camargo y Venegas-Martínez (2003). 
$x_{1}, x_{2}$ y $x_{3}$ рог los correspondientes márgenes iniciales $\mathrm{y}$, en su caso, por el margen adicional cuando la calidad crediticia del inversionista así lo requiera, considerando los "spreads" o posiciones opuestas que se generen. En México, la cámara de compensación y liquidación de la bolsa de futuros es un fideicomiso. En este caso, los margenes iniciales son llamados aportaciones iniciales mínimas y los margenes adicionales aportaciones excedentes.

Vamos a decir que los contratos futuros $F=\left\{F_{t, T_{1}}^{(n)}, F_{t, T_{2}}^{(n)}, F_{t, T_{3}}^{(n)}\right\}$ con fechas de vencimiento $T_{1}, T_{2}$ y $T_{3}$, en cantidades $x_{1}, x_{2}$ y $x_{3}$ cubren a $f$ si $F$ y $x_{1}, x_{2}$ y $x_{3}$ satisfacen el sistema de ecuaciones previamente establecido. Es importante señalar algunas limitaciones del método. Primero, cuando un mercado dispone de cuatro o más series de futuros de dólar, se obtendría un sistema de tres ecuaciones con cuatro o más incógnitas. Por lo tanto, existe un número infinito de estrategias de cobertura, de las cuales se pueden escoger algunas que cumplan con atributos deseables como es la liquidez de los futuros. Segundo, en el caso mexicano, el método cubre de manera limitada contra fluctuaciones en el tipo de cambio, el rebalanceo periódico de la estrategia permitirá una mejor protección. El método supone liquidez infinita (efecto precio despreciable, en el sentido de que se mantienen las relaciones de arbitraje entre futuros y contado aunque se aumente el tamaño de la transacción) y, por último, supone que se pueden vender futuros por cantidades divisibles. Sin embargo, es importante notar que la estandarización de los contratos, no permite tomar posiciones sobre nominales distintos a los múltiplos generados por el tamaño del contrato.

\section{Recomendaciones generales para el control de riesgos}

A continuación se presenta, en forma breve, una guía para la conducción de la administración de riesgos financieros que requiere el país:

1) Toda institución financiera debe mantener políticas y procedimientos robustos que consideren el uso de productos derivados.

2) Cambios significativos en el manejo de nuevos derivados deberán ser evaluados y, en caso de su instrumentación, deben ser cubiertos en forma adecuada. Las posiciones en exceso deben recibir una pronta atención.

\subsection{El proceso de cuantificación de riesgos}

A continuación se presenta un conjunto de recomendaciones con respecto al proceso de cuantificación de riesgos.

1) Los riesgos deben ser cuantificados en forma períodica para todas las actividades de la institución.

2) Los estándares de medición de riesgos deben ser entendidos por el personal de todos los niveles de la institución, y deben proporcionar una estructura común para monitorear y limitar el riesgo asumido en todas las actividades.

3) El análisis de situaciones extremas, no debe ser limitado a un ejercicio cuantitativo que calcule las pérdidas o ganancias potenciales, sino que debe también incluir las acciones a tomar sobre ciertos escenarios. 


\subsection{Responsabilidad de las autoridades}

Finalmente, se presentan las actividades y acciones que las autoridades financieras deben llevar a cabo:

1) La autoridad reguladora debe verificar que las entidades participantes en el sector cuenten con sistemas de administración de riesgos adecuados a su volumen de operación.

2) Los administradores de riesgos deben contar con una certificación, que respalde su capacidad técnica, expedida por la autoridad reguladora.

\section{Conclusiones}

En este trabajo se ha presentado un diagnóstico y una estrategia en cuanto a los cambios de actitud y tecnológicos que requiere la administración de riesgos en las instituciones financieras del país. Hasta ahora, el cambio tecnológico que demanda la administración de riesgos en México se queda corto en cuanto a las necesidades y expectativas de cambio del entorno. La ausencia tan prolongada de mercados de instrumentos financieros de cobertura, nos invita a evaluar sus efectos sobre el sector financiero mexicano. En particular, en el episodio de diciembre de 1994, llama la atención la enorme exposición a diferentes tipos de riesgos y la imposibilidad de administrarlos a falta de un mercado de coberturas contra contingencias financieras, situación que no permitió a los agentes económicos planear adecuada y oportunamente sus recursos en el corto y largo plazo (véase Venegas-Martínez, 2001a).

La administración de riesgos tiene que ir más allá de la simple interpretación de resultados del "software" comercial especializado, se requiere de administradores de riesgos comprometidos con el cambio, específicamente con el cambio de paradigmas y con el cambio tecnológico. Contar con sistemas eficientes y congruentes con nuestra realidad contribuirá, en cierta medida, a generar un ambiente de estabilidad en la economía nacional, pues cuando estos sistemas se usan adecuadamente protegen a los agentes económicos contra fluctuaciones adversas en los mercades, modificando en forma positiva las expectativas dentro de la economía en el mediano y largo plazo.

La transformación del sistema financiero mexicano hacia una administración de riesgos eficiente es, a todas luces, una prioridad, antes de que se repita otra debacle financiera como la de 1994-1995, periodo en donde la exposición a los riesgos de mercado y de crédito alcanzaron niveles preocupantes y las medidas de cobertura fueron mínimas o nulas, el desenlace todos lo conocemos: un sector financiero que muestra todavía rezagos de la crisis. 


\section{Bibliografía}

González-Arechiga, B., Venegas-Martínez, F. y J. Díaz Tinoco, (2000). Riesgo de tasas de interés e inmunización por duración y convexidad con futuros: análisis local y de valor en riesgo. Investigación Económica, Fac. de Economía, UNAM, Vol. 60(233), pp. 72-112.

Islas Camargo, A. y Venegas-Martínez, F. (2003). Pricing derivatives securities with prior information on long-memory volatility. Economía Mexicana, Nueva Epoca, CIDE, 12, por aparecer.

Venegas-Martínez, F. (2002). Cobertura de flujos financieros con instrumentos de renta fija, Estudios Económicos, COLMEX, 17(2), pp. 171-192, 2002.

Venegas-Martínez, F. (2001). Una guía completa para economistas en la valuación de opciones. Gaceta de Economía, ITAM, Año 6, No. 12, pp. 155-212.

Venegas-Martínez, F. (2001a). Temporary stabilization: a stochastic analysis. Journal of Economic Dynamics and Control, Vol. 25(9), September, pp. 1429-1449.

Venegas-Martínez, F. y Dubcovsky, G. (2002). Retrospectiva y Perspectiva de la Administración de Riesgos Financieros en Mexico, Ejecutivos de Finanzas, IMEF, 31(8), pp. 153-168. 